\title{
Adaptación del nuevo grado de Medicina al Espacio Europeo de Educación Superior. ¿Cuál ha sido la aportación de Bolonia?
}

\author{
Francisco Arnalich Fernández
}

\section{El Espacio Europeo de Educación Superior}

La creación del llamado 'Espacio Europeo de Educación Superior' (EEES) tiene su origen en la Declaración de Bolonia (19 de junio de 1999); es una iniciativa para reformar el sistema educativo europeo, que fue suscrita por los 29 países que conformaban entonces la Unión Europea, a los que se han ido añadiendo otros pertenecientes al Espacio Europeo de Libre Comercio y del este y el centro de Europa, hasta un total de 47 países. Entre sus objetivos destacan los siguientes:

- Reforzar la competitividad de la educación europea superior.

- Favorecer la tasa de empleo y movilidad de los universitarios.

- Adoptar un sistema comprensible y comparable de estudios.

- Establecer un sistema de créditos como unidad de medida de carga docente-European Credit Transfer System (ECTS)-.

- Promocionar la movilidad y el intercambio de estudiantes, profesores y profesionales posgraduados entre los países.

A estos objetivos, se añadieron en las reuniones de Praga (2001) y Berlín (2003) los tres objetivos siguientes:

- Integrar el aprendizaje de por vida (life-long learning) en la estrategia educativa global.
- Implicar a los estudiantes en el desarrollo del proceso.

- Promover la creación de un área europea en investigación.

Después se han seguido manteniendo reuniones bianuales (Bergen 2005, Londres 2007, Lovaina 2009, Budapest-Viena 2010) y las próxima reunión se desarrollará en Bucarest (2012).

Desde el punto de vista legislativo, se inicia la reforma educativa a través de la Ley Orgánica de Modificación de la Ley Orgánica de Universidades (LOMLOU), que es la que da cobertura legal y formal al proceso de Bolonia en España, puesto que en la anterior Ley Orgánica de las Universidades (LOU, 2001) no se tuvo en cuenta el proceso de Bolonia, y culmina con el Real Decreto (1393/2007, de 29 de octubre), por el que se establece la ordenación de las enseñanzas universitarias oficiales y se indica que en el curso académico 2010-2011 no podrán ofertarse plazas de nuevo ingreso en primer curso para las actuales titulaciones de licenciado.

Sin embargo, para alcanzar una significativa mejora de la calidad de los estudios universitarios en nuestro país quizá fuera preciso ajustar y priorizar la oferta educativa, que todavía sigue siendo extraordinariamente amplia y diversa, como indica la magnitud de la oferta global de plazas de nuevo ingreso en la universidad, que asciende a 336.165 plazas, cifra ligeramente inferior a la del año pasado (341.332), distribuidas entre 2.387 grados y 3.089 másteres.
Catedrático de Medicina Interna. Hospital Universitario La Paz. UAM. Comisión de Evaluación para la Verificación de Títulos de Grado de Ciencias de la Salud. Agencia Nacional de Evaluación de la Calidad y Acreditación (ANECA).

E-mail

francisco.arnalich@uam.es 


\section{Implantación del proceso \\ de convergencia europea en los estudios de medicina}

Un año después, en cumplimiento de la disposición adicional novena de este decreto, el Ministerio de Educación y Ciencia (MEC) publica una orden ministerial (Orden ECI/332/2008, de 13 de febrero), por la que se establecen los requisitos para la verificación de los títulos universitarios oficiales que habiliten para el ejercicio de la profesión de médico. En el anexo de esta orden se detalla la estructura del nuevo grado de Medicina, siguiendo las directrices del Libro Blanco de ANECA. De este modo, la orden ministerial se convierte en la norma obligada sobre la que se redactan los planes de estudio de los nuevos grados de Medicina.

En el anexo de esta orden se detallan los objetivos del nuevo grado, aunque son formulados en realidad como un conjunto de 37 'competencias específicas' más que objetivos propiamente dichos. De este modo se recogen las 34 competencias definidas por el Libro Blanco de la Titulación de Medicina (Conferencia Nacional de Decanos de Facultades de Medicina Españolas, 20 de abril de 2005), a lo que se añaden otras tres competencias adicionales. Además, se detalla la estructura del plan de estudios, que debe incluir como mínimo los cinco módulos siguientes:

- Morfología, estructura y función del cuerpo humano (64 ECTS).

- Medicina social, habilidades de comunicación e iniciación a la investigación (30 ECTS).

- Formación clínica humana (100 ECTS).

- Procedimientos diagnósticos y terapéuticos (40 ECTS).

- Prácticas tuteladas y trabajo de fin de grado (60 ECTS).

Se reservan los restantes 30 créditos para la oferta de 30 créditos en materias optativas, y entre ellas los estudiantes podrán obtener un reconocimiento académico de 6 créditos por la participación en actividades universitarias culturales, deportivas, de representación estudiantil, solidarias y de cooperación.

Junto al proceso de implantación del grado de Medicina, conforme a los principios de la convergencia europea, se ha emprendido una po- lítica de incremento en el número de las plazas ofertadas para iniciar los estudios de medicina, de forma no consensuada, en opinión de los decanos de las facultades de medicina ('Bolonia: los mismos medios, menos libertad de cátedra y más burocracia. Diario Médico, 21 de abril de 2009). El MEC, según ha expresado el profesor Gabilondo, ha admitido que desde el curso 20062007 se ha producido un incremento de plazas del $41,2 \%$, de forma que se podrá cumplir en cuatro años el compromiso firmado con Sanidad de crear 2.000 plazas nuevas de medicina en cinco años. Actualmente existen 39 facultades de medicina en España, 31 públicas y 8 privadas (frente a las 27 facultades existentes en el 2005), y el número de plazas para estudiar medicina crece un $7,1 \%$. El próximo curso $2010-2011$ podrán empezar esta carrera 6.673 alumnos, frente a los 6.229 de este curso. La única comunidad autónoma que reduce su oferta de grado es Cataluña, donde siete de sus centros no amplían ningún puesto y la facultad de la Universitat de Barcelona los reduce un 4,07\%. Los incrementos más significativos, al menos en el borrador inicial de adjudicaciones, se dan en Córdoba (20,83\%), La Laguna (15,38\%), Santiago de Compostela $(14,29 \%)$, Santander y Universidad Rey Juan Carlos, de Madrid (11,11\%). Por comunidades autónomas, Madrid (1.291) y Andalucía (1.145) lideran la oferta de plazas para el próximo curso, y entre las dos suman el $35,15 \%$ del total de puestos.

\section{Aportaciones de la reforma del plan de estudios de medicina}

\section{Adquisición de 'competencia clínica' como principal objetivo docente}

El objetivo específico del grado de Medicina es la formación de profesionales capaces de indicar y realizar actividades dirigidas a la promoción, mantenimiento y recuperación de la salud. De este modo, el nuevo rol de las facultades de medicina será (Fig. 1):

- Enseñar el conocimiento, habilidades y actitudes que necesitará el estudiante para practicar la medicina.

- Certificar que cada graduado es clínicamente competente. 


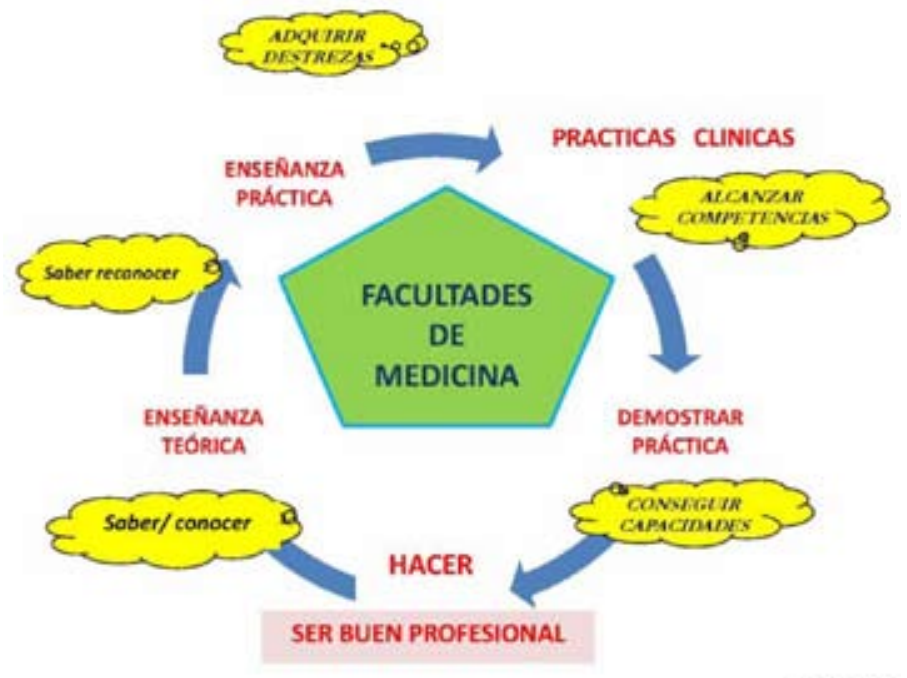

F. Arnalich

Figura 1. El nuevo rol de las facultades de medicina después de la adaptación al proceso de Bolonia.

Competencia clínica es el conjunto de conocimientos, habilidades, destrezas, actitudes y valores necesarios para la ejecución de acciones relacionadas con la prevención, diagnóstico y tratamiento de las enfermedades, e igualmente necesarios para la interacción interpersonal (pacientes, familiares, otros profesionales) en la búsqueda de soluciones a los graves problemas de salud. Dicho de otra manera, la competencia clínica requiere el dominio de conocimientos específicos, propios de cada patología, de habilidades de comunicación y de organización, de habilidades y destrezas para trabajar en equipo y para solucionar problemas, y de habilidades para el razonamiento en función de las evidencias encontradas. Igualmente engloba valores tales como la vocación de servicio, la sensibilidad social, la responsabilidad, el compromiso, la empatía... (Fig. 2).

\section{Un primer año bien definido (60 créditos) de formación básica}

Esta formación es común y obligatoria para todas las facultades de medicina. El 60\% de la carga docente (36 ECTS) se configura por materias básicas comunes a los diferentes grados de la rama de Ciencias de la Salud (Anatomía Animal, Anatomía Humana, Biología, Bioquímica, Estadística, Física, Fisiología, Psicología) o por otras materias siempre que se justifique su carácter básico para la formación inicial del estudiante o su carácter transversal. De este modo se facilita el reconocimiento de créditos cursados en otros títulos (Enfermería, Fisioterapia, Farmacia, Veterinaria, etc.) al estudiante que cambia su orientación y solicita el traslado al grado de Medicina.

\section{Descripción objetiva y comparable de cada módulo y materia del plan de estudios}

Incluye la enumeración de las competencias que adquiere el estudiante en cada módulo o materia, una breve descripción de sus contenidos y su equivalencia de carga docente en número de créditos, los diferentes tipos de actividades formativas previstas, así como la metodología de enseñanza-aprendizaje y su relación con las competencias que debe adquirir el estudiante. También se define el sistema de evaluación de la adquisición de las competencias y el sistema de calificaciones.

\section{Inclusión de prácticas externas obligatorias (54 ECTS) y trabajo de fin de grado}

Se desarrollan en los últimos semestres del grado, preferentemente durante el sexto año (Tabla). Consta de estancias tuteladas en hospitales y centros de atención primaria, de manera que 


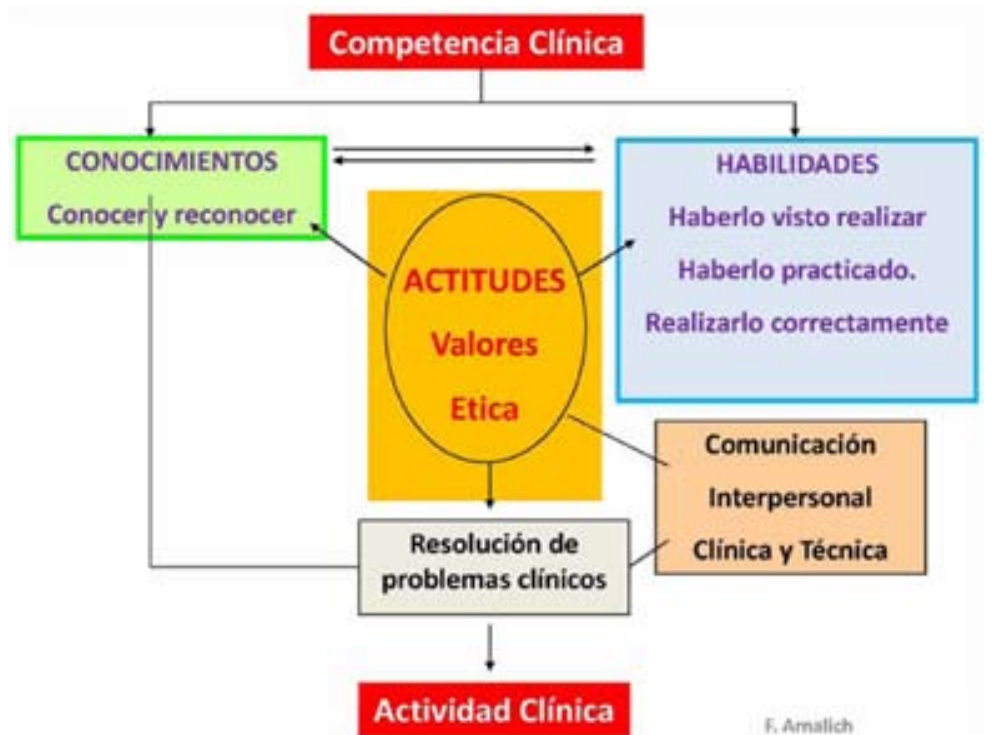

Figura 2. Componentes de la competencia clínica.

el estudiante adquiera experiencia clínica en seis áreas distintas. Presenta las siguiente competencias específicas:

- Aplicar los conocimientos, desarrollar habilidades clínicas transversales, trabajar en equipo multiprofesional.

- Adquirir capacidad para relacionar las diferentes materias con el fin de alcanzar un conocimiento más integrador

\section{Descripción del personal académico y recursos materiales disponibles}

También se enumeran las instalaciones (espacios, aulas, laboratorios, equipamiento científico y técnico, biblioteca, salas de lectura, nuevas tecnologías, etc.), y los hospitales y centros sanitarios concertados para la docencia práctica, que reúnen los requisitos legales para su acreditación (Orden de 31 de julio de 1987, que establece las bases generales del régimen de conciertos entre las universidades y las instituciones sanitarias). Esta información no había sido ofrecida por las universidades en los anteriores planes de estudio y supone un compromiso de adecuación entre capacidad docente y oferta lectiva para garantizar el desarrollo de las actividades formativas planificadas.

\section{Sistema de garantía de la calidad}

La información habitualmente se refiere a un sistema general de la universidad o del centro responsable de las enseñanzas aplicable al título.

\section{Limitaciones}

Existen una serie de aspectos mal contemplados en la actual reforma, que ya es vigente para este curso académico en todas las facultades de medicina. Entre ellos, señalemos la falta de medios económicos para una enseñanza más individualizada y las dificultades para implementar una renovada enseñanza práctica, que utilice los nuevos instrumentos de evaluación capaces de medir el nivel de competencia clínica alcanzado por el alumno en los sucesivos módulos y, muy especialmente, en las prácticas externas tuteladas.

En resumen, la reforma curricular de los estudios de Medicina adaptados a Bolonia no es sino el principio de un largo camino por recorrer orientado a la mejora de la competencia profesional de los titulados. Es tiempo, pues, de innovar. Atrás debe quedar esa metodología docente obsoleta basada en la transmisión de datos y en su memorización. Innovar supone provocar ad- 
Tabla. Características específicas del módulo de prácticas externas tuteladas (total: 60 ECTS).

\begin{tabular}{lcccc}
\hline Materias & ECTS & Carácter & Curso & Unidad temporal \\
\hline Clínica práctica & 54 & Obligatorio & $3 .^{\circ-60^{\circ}}$ & Semestral/Anual \\
\hline Trabajo fin de grado & 6 & Obligatorio & $60^{\circ}$ & Anual \\
\hline
\end{tabular}

miración, al atreverse a imaginar soluciones no vistas antes. Como solía decir el maestro Antoni Gaudí, 'la originalidad consiste en el retorno al origen'. De acuerdo con esta idea, podríamos asumir que la verdadera innovación en el grado de Medicina quizá pase por el regreso a los fundamentos del proceso educativo, es decir, a la transmisión directa del saber mediante la relación estrecha entre profesor y alumno a la cabecera del paciente, día a día.

Por lo tanto, se puede afirmar que después de este año seguirá existiendo la necesidad de más Bolonia. ¡Gracias, Bolonia!

\section{Bibliografía}

1. Christensen L. The Bologna process and medical education. Med Teach 2004; 26: 625-9.
2. Global Minimum Essential Requirements in Medical Education. Institute for International Medical Education Core Committee. Med Teach 2002; 24: 130-5.

3. Miller GE. The assessment of clinical skills/competence/ performance. Acad Med 1992; 65 (Suppl): S63-7.

4. Epstein R, Hundert E. Defining and assessing professional competence. JAMA 2002; 287: 226-35.

5. Kronfly E, Ricarte JI, Juncosa S, Martínez-Carretero JM. Evaluación de la competencia clínica de las facultades de medicina de Cataluña, 1994-2006: evolución de los formatos de examen hasta la evaluación clínica objetiva y estructurada (ECOE). Med Clin (Barc) 2007; 129 : 777-84.

6. Lobato RD, Lagares A, Alén JF, Alday R. El desarrollo del proceso de 'Bolonia’ y el Grado de Medicina. Situación actual y expectativas para su implantación definitiva. Neurocirugía 2010; 21: 146-56.

7. Oriol A. Plan Bolonia: 'mucha mística y pocos recursos' Diario Médico (Suplemento Especial Formación), 28 de enero de 2010; p. 12. 